\title{
A longitudinal study on the persistence of Livestock Associated-MRSA in swine herds
}

van der Wolf, P.J. ${ }^{1 *}$

Broens, E.M. ${ }^{2}$, Köck, R. ${ }^{3}$, Graat, E.A.M. ${ }^{2}$

1 Pig Health Department, Animal Health Service (AHS), Deventer, The Netherlands.

2 Quantitative Veterinary Epidemiology Group, Wageningen Institute of Animal Sciences, Wageningen University, Wageningen, The Netherlands.

3 Institute of Hygiene, University Hospital Münster, Münster, Germany

P. van der Wolf, Pig Health Department, Animal Health Service, P.O.Box 9, 7400 AA Deventer, The Netherlands, e-mail: p.vd.wolf_at_gddeventer. com, fax: +31-570-634104

\begin{abstract}
In recent years, a new type of MRSA, now called livestock-associated MRSA (LA-MRSA), belonging to the clonal complex (CC) 398, has globally emerged in swine world wide. Aim of this study was to gain more insight into the persistence of LA-MRSA in different types of pig farms over a period of two years. To investigate this, 15 MRSA-positive herds from a previous study were selected; an additional pig farm was selected because this one was found to be positive with a human MRSA-strain. Starting in September 2009, five dust samples were collected every two months. Samples were analysed for MRSA and spa-typing was done to confirm that MRSA isolates belonged to CC398 and to gain insight into persistence of strains within a farm.

Three herds were positive on all sampling occasions and were consistently contaminated with LA-MRSA. In the remaining herds, occasionally no positive dust samples were found at some sampling moments. The predominating spa types were †0 11 and +108 . A maximum of 5 different spa-types were found in two herds, with 3 different spa-types present in one sampling, indicating multiple introductions.

These results show that LA-MRSA remains present on a pig farm over a long period. Most likely, transmission within the herd occurs after initial introduction, and an endemic situation seems to be the endpoint. The relatively low sensitivity of dust sampling compared to sampling of animals, the small sample size and lack of strict standardization of dust sampling might explain occasional negative samplings in overall positive herds. However, a true change of a positive MRSA-status to a negative status, followed by re-introduction cannot be ruled out in our study design.
\end{abstract}

\section{Introduction}

Since the first detection of a new type of MRSA in pigs in 2004 (Voss et al., 2005) belonging to the clonal complex (CC) 398, as determined by multilocus sequence typing (MLST), this MRSA clone has globally emerged in swine and other livestock worldwide (Broens et al., 2008) (Livestock-associated MRSA, LA-MRSA)Observational studies have been limited to single observations at farm level so far, and information on the transmission and persistence of LA-MRSA within a farm is very limited. To develop effective intervention strategies, more insight is needed into persistence of LA-MRSA within a pig farm over a longer time period. Persistence of LA-MRSA might differ depending on management strategies on a farm, which are very different between farm types. For example, the all in-all out production system is often applied on finishing farms, whereas a continuous production system is usually applied on farrowing farms.

The study objective was to gain more insight into the persistence of LA-MRSA in different types of pig farms over a period of two years.

\section{Material and Methods}

Fifteen pig farms which were found positive for MRSA CC398 in a previous prevalence study on Dutch pig farms (Broens et al., submitted), were selected comprising five finishing, farrow-to-finish and farrowing farms, respectively. One particular pig farm was added, because this one was found to be positive with a human MRSA-strain (spa-type t002/ST5). On all farms, five dust samples (s 1 kit ringer solution, Sodibox) were collected every two months from five different farm sections to sample all age categories present. No more than one sample was collected in each compartment. At the start of the study, samples were taken by an employee of the Animal Health Service and the farmer was instructed how to take the samples. Information on farm characteristics was collected by a questionnaire. After that, a parcel containing sampling materials, a submission form, a short questionnaire, a billing form and materials for return posting was sent to the farmers 
every two months. The farmers received payment for each sampling submitted. Questionnaires were checked for completeness at receipt. Sampling started in September 2009 and will run until September 2011 ; results from samplings till March 2011 are analysed and presented in this paper.

MRSA isolation was performed on single dust samples according to the protocol described by Broens et al. (201 1a) at the Animal Health Service, The Netherlands. One suspected colony per sample was confirmed to be MRSA by 2 PCR tests for the S. aureus specific DNA-fragment (de Neeling et al., 1998) and the mecA gene (Martineau et al., 1998). For all MRSA isolates spa typing was performed at the Institute for Hygiene, Germany (Harmsen et al., 2003). So far, spa-typing was only done on samples taken before November 2010. A farms was classified MRSA-positive if at least one dust sample tested MRSA-positive at a sampling.

\section{Results}

So far, 4-10 samplings were received and analysed per farm, resulting in 677 samples. Farm 7 stopped farming during the study, so no samples were received from this farm after sampling 4. Farm 8 did not comply with the sampling scheme and sent in samples very irregularly. One sampling from farm 5 was lost in the mail. One dust sample was missing in samplings from farm 8, farm 12 and farm 13. Average time between samplings was 9 weeks (SD=3.7 weeks), varied from less than a week (farm 8) to 24 weeks (farm 5) and was not different for the different herd types ( $P=0.86)$. Of all samples, 220 were positive $(32.5 \%)$, and was lower for farrow-to-finish farms $(25.0 \%$; $P=0.01)$ than for farrowing farms $(35.8 \%)$ and finishing farms (36.6\%). The average percentage of positive dust samples per farm over the study period was $34 \%$, ranging between 10 and $62 \%$, and was not different per herd type, being resp. $37.2 \%$ for farrowing, 26.4 for farrow-to-finish and $37.0 \%$ for finishing farms $(\mathrm{P}=0.49)$. In only 6 out of 136 samplings, all 5 dust samples were positive. At least one dust sample was MRSA-positive in $72.1 \%(98 / 136)$ of samplings moments. Per farm, on average $74 \%$ of sampling moments was found positive, varying from 38\% (3 out 8 samplings) to 100\% (farm 1, 7 and 15) where at all samplings MRSA-positive samples were found. This percentage was $80.4 \%, 68.9 \%$ and $66.7 \%$ for farrowing, farrow-tofinish and finishing farms $(\mathrm{P}=\mathrm{O} .30)$, respectively.

So far, spa-typing was done on 181 isolates out of 220 positive samples. Fourteen spa-types were identified; 3 formerly confirmed to be non-CC398 (†002, †1 27 and †2 164), 9 formerly confirmed to be CC398 (EFSA, 2009; Reischl et al., 2009; Wagenaar et al., 2009), and 2 'new' spa-types most probably belonging to CC398 (t6320 and t7621; Table 1). Spa-type 1011 and +108 accounted for $73.5 \%$ of all spa-types. On five farms, only 1 spa-type was isolated over the whole study period, these were spa-type t1 08 in 3 farms and spa-types t899 and t0 11 in one farm each. On the remaining 11 farms, 2 to 5 different spa-types were found over the study period and up to 3 different spa-types at one sampling moment. Farm 16, which was selected for the presence of a human MRSA-strain (spa-type t002), was MRSA-positive in sampling $1-4$ and 8 . In sampling 3, 4 and 8, spa-type t002 was again found and in sampling 1, spa-type t2 164 was found; typing results of sampling 2 were missing.

Table 1. Spa-types, their repeat succession and their frequencies (number and percentage) found on 16 pig herds.

\begin{tabular}{|c|c|c|c|}
\hline \multirow[t]{2}{*}{ spa -type } & \multirow[t]{2}{*}{ repeat succession } & \multicolumn{2}{|c|}{ frequency } \\
\hline & & $\mathrm{n}$ & $\%$ \\
\hline 1011 & $08 \cdot 16.02 \cdot 25 \cdot 34 \cdot 24 \cdot 25$ & 86 & 47.5 \\
\hline $\mathrm{t} 10 \mathrm{~s}$ & $08-16-02-25-24-25$ & 47 & 26 \\
\hline 1899 & $07-16-23-02-34$ & 18 & 9.9 \\
\hline $1127^{\bullet}$ & 07.23 .21 .16 .34 .73 .13 & 6 & 33 \\
\hline $16320^{*}$ & $08-16-02-25-34-24-34-24-25$ & 5 & 2.8 \\
\hline 1034 & $08.16 \cdot 02.25 .02 .25 \cdot 34.24 .25$ & 4 & 2.2 \\
\hline $1762 t^{\prime}$ & $08-16-02-25-34-02-25-34-24-25-25$ & 4 & 2.2 \\
\hline $1002^{*}$ & $26-23-17-34-17-20-17-12-17-16$ & 3 & 1.7 \\
\hline 12346 & $08-16-02-25-34-24-24-25$ & 3 & 1.7 \\
\hline 11184 & $08-16-02-25-25$ & 1 & 0.6 \\
\hline 11451 & $08-16-02-25-34-25$ & 1 & 0.6 \\
\hline 11606 & $08-16-34-34-24-25$ & 1 & 0.6 \\
\hline $12164^{*}$ & $26 \cdot 23 \cdot 17 \cdot 34 \cdot 17 \cdot 82 \cdot 17 \cdot 12 \cdot 17 \cdot 16$ & 1 & 0.6 \\
\hline 12330 & $08 \cdot 16 \cdot 02 \cdot 25 \cdot 34 \cdot 24 \cdot 25 \cdot 25$ & 1 & 0.6 \\
\hline
\end{tabular}

*non-CC398; \#'new' spa-type, unknown CC 


\section{Discussion}

These data show that pig farms can remain MRSA-positive for over a year, and that the same spa-type can be found on a farm during this period. This holds for farrowing, farrow-to-finish and finishing farms. Whether the discriminatory power of spa-typing method is sufficient to indicate continuous contamination of a farm by the same MRSA-strain, needs confirmation by further genetic typing of the isolates using whole genome techniques. For spa-typing, a single locus, the staphylococcal protein A, is sequenced. Despite a high concordance of results from spa-typing with other typing methods such as MLST, the spa-type only indicates that isolates belong to a certain Clonal Complex. Consequently, selected isolates will have to be typed by MLST to confirm that the strains belong to CC398. Selecting more than one suspected colony per sample will also contribute to getting more insight in continuous contamination or re-introduction. Most likely, transmission within the herd occurs after initial introduction, and an endemic situation seems to be the endpoint.

The relatively low sensitivity of dust sampling compared to sampling of animals, the small sample size and lack of strict standardization of dust sampling might explain the, occasionally found, negative samplings in overall positive herds (Broens et al., 201 la). However, a true change of positive MRSA-status to a negative status, followed by re-introduction cannot be ruled out in our study design. On the other hand, repeatedly isolating the same spa-type suggests that the herd is still contaminated with the same MRSA-strain, but sometimes at levels below the detection limit of the method we used. The repeated isolation of spa-type t002 in one herd shows that also human MRSA strains can remain in a herd for a period of more than a year.

The occurrence of more than one spa-type in some herds might indicate that more than one introduction has taken place in that herd. This can be caused by buying MRSA-positive replacement stock from different sources or by a lacking external biosecurity of the farm (Broens et al., 2011 b). Identification and sampling of contact farms in the national identification and registration system might help to confirm the introduction of MRSA by the purchase of pigs.

\section{Conclusions}

Pig herds can remain MRSA-positive for over a year, not only with livestock-associated MRSA-strains, but also with a human MRSA-strain. It can be concluded that spontaneous extinction of MRSA from a pig population does not occur frequently. This indicates that control strategies should focus on preventing introduction of MRSA into a farm and that active intervention measures are needed to eradicate MRSA from pig farms. Further research in needed to determine which intervention measures are effective.

\section{Acknowledgements}

The authors thank the farmers for their kind cooperation.

The authors thank Ms M. Meijerink and Mrs I. Horsman-Schriemer for their assistance with the collection of the samples and keeping the farmers motivated to participate in this project.

This work is co-financed by the INTERREG IV A Germany-Netherlands programme through the EU funding from the European Regional Development Fund (ERDF), the Ministry for Economic Affairs, Energy, Building, Housing and Transport of the State of North-Rhine Westphalia, the Ministry of Economic Affairs, Labour and Traffic of Lower Saxony, the Dutch Ministry of Economic Affairs, Agriculture and Innovation, the Product Boards for Livestock, Meat and Eggs, the Food Safety Authority in The Netherlands, and the Provinces of Friesland, Groningen, Drenthe, Overijssel, Gelderland, Noord-Brabant and Limburg of The Netherlands. This project is coordinated by the EU-region Rhine-Waal and managed by GIQS

\section{References}

Broens, E.M., Graat, E.A.M., Engel, B., van Oosterom, R.A.A., van de Giessen, A.W., van der Wolf, P.J., 201 1a, Comparison of sampling methods used for MRSA-classification of herds with breeding pigs. Vet. Microbiol. 147, 440-444. Broens, E.M., Graat E.A.M., van der Wolf, P.J., van de Giessen, A.W., van Duijkeren E., Wagenaar J.A., van Nes, A., Mevius, D.J., de Jong, M.C.M., 201 1b, MRSA CC398 in the pig production chain. Prev. Vet. Med. 98, 182-189. Broens, E.M., Van Cleef, B., Graat, E.A., Kluytmans, J.A., 2008, Transmission of methicillin-resistant Staphylococcus aureus from food production animals to humans: a review. CAB Reviews3, 12.

De Neeling, A.J., van Leeuwen, W.J., Schouls, L.M., Schot, C.S., Veen-Rutgers, A., Beunders, A.J., Buiting, A.G., Hol, C., Ligtvoet, E.E., Petit, P.L., Sabbe, L.J., van Griethuysen, A.J., van Embden, J.D., 1998, Resistance of staphylococci in The Netherlands: surveillance by an electronic network during 1989-1995. J.Antimicrob. Chemother. 41, 93-101.

EFSA, 2009, Analysis of the baseline-survey on the prevalence of methicillin-resistant Staphylococcus aureus (MRSA) in holdings with breeding pigs, in the EU, 2008. Part A: MRSA prevalence estimates; on request of the European Commis- 
sion, EFSA J. 7, 1376. Available online at http://www.efsa.europe.eu.

Harmsen, D., Claus, H., Witte, W., Rothganger, J., Turnwald, D., Vogel, U., 2003, Typing of methicillin-resistant Staphylococcus aureus in a university hospital setting by using novel software for spa repeat determination and database management. J. Clin. Microbiol. 41, 5442-5448.

Martineau, F., Picard, F.J., Roy, P.H., Ouellette, M., Bergeron, M.G., 1998, Species-specific and ubiquitous-DNA-based assays for rapid identification of Staphylococcus aureus. J. Clin. Microbiol. 36, 618-623.

Reischl, U., Frick, J., Hoermansdorfer, S., Melzl, H., Bollwien, M., Linde, H.J., Becker, K., Köck, R., Tuschak, C., Busch, U., Sing, A., 2009, Single-nucleotide polymorphism in the SCCmec-orfX junction distinguishes between livestock-associated MRSA CC398 and human epidemic MRSA strains. Eurosurveillance 14. Available online at www.eurosurveillance. org

Voss, A., Loeffen, F., Bakker, J., Klaassen, C., Wulf, M., 2005, Methicillin-resistant Staphylococcus aureus in pig farming. Emerg. Inf. Dis. 11, 1965-1966.

Wagenaar J.A., Giessen van de A.W., 2009, Veegerelateerde MRSA: epidemiologie in dierlijke productieketens, transmissie naar de mens en karakterisatie van de kloon, RIVM-report 33022400 1. In Dutch with a summary in English. Available online at http://www.rivm.nl/bibliotheek/rapporten/330224001.pdf 